\title{
Serious complication following use of StomaphyX
}

Roux-en-Y gastric bypass (RYGB) is a popular surgical procedure to treat morbid obesity [1]. StomaphyX (endoGastric Solutions Inc., Redmond, Washington, USA) is designed for incisionless transoral endoscopic plication and revision of the gastric pouch (EPRGP) in case of pouch dilation with weight gain [2,3]. Nonresorbable polypropylene fasteners create fullthickness plications of the pouch wall, reducing its volume. StomaphyX was cleared by the Food and Drug Administration (FDA) in April 2007 [4] and clinical results are becoming available, with only $3 \%$ early complications [5].

We present the case of a 38-year-old woman who underwent StomaphyX EPRGP in 2007, after RYGB in 2000. The procedure was carried out in a peripheral hospital by a surgeon and was not planned within the regulations governing ongoing clinical trials. After 3 days, the patient was referred to our hospital with fever and thoracic pain. Thoracic X-ray showed left lung empyema ( $\bullet$ Fig. 1). Antibiotics were started and she underwent two thoracic surgical procedures to drain the left hemithorax and to remove the pleural membranes ( Fig. 2). Upper gastrointestinal endoscopy showed three StomaphyX fasteners through the distal oesophageal wall and a dilated gastric pouch with another three fasteners around the gastrojejunostomy ( Fig. 3 ). Because of persistent empyema, a third draining thoracotomy was carried out 14 days later, which revealed adhesions at the distal esophagus. Streptococcus viridans and non-aureus Staphylococcus were cultured and antibiotics started. After a 28-day stay in the hospital, the patient was fit enough to be discharged. At present, 4 years after the complicated StomaphyX procedure, the patient is still under treatment for postoperative neuralgic pain of the left hemithorax. She was not covered by a clinical trial insurance. This case report illustrates the risk of serious complications of StomaphyX and the ethical implications of using new devices outside the control of regulated clinical trials.

Endoscopy_UCTN_Code_CPL_1AH_2AJ

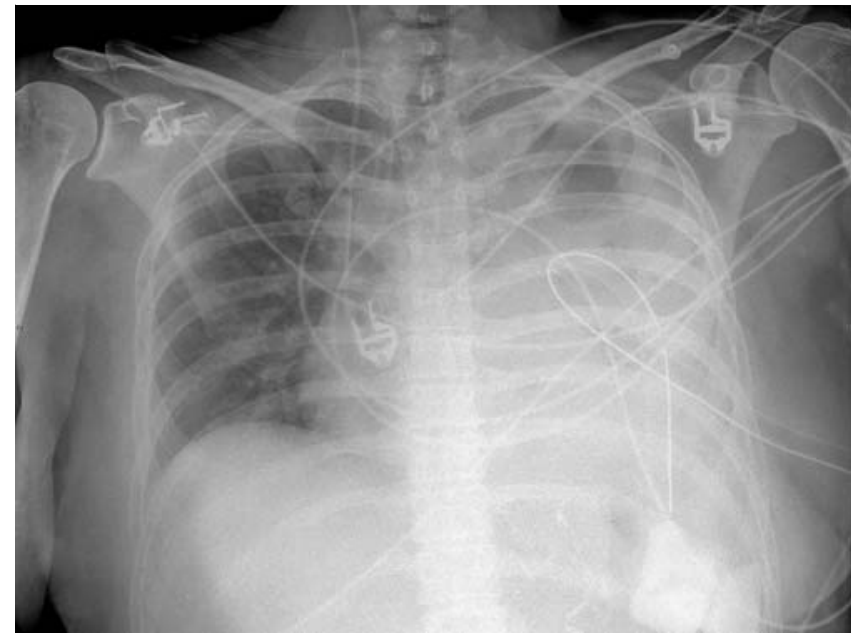

Fig. 1 Chest X-ray showing left lung empyema with a thoracic drain.

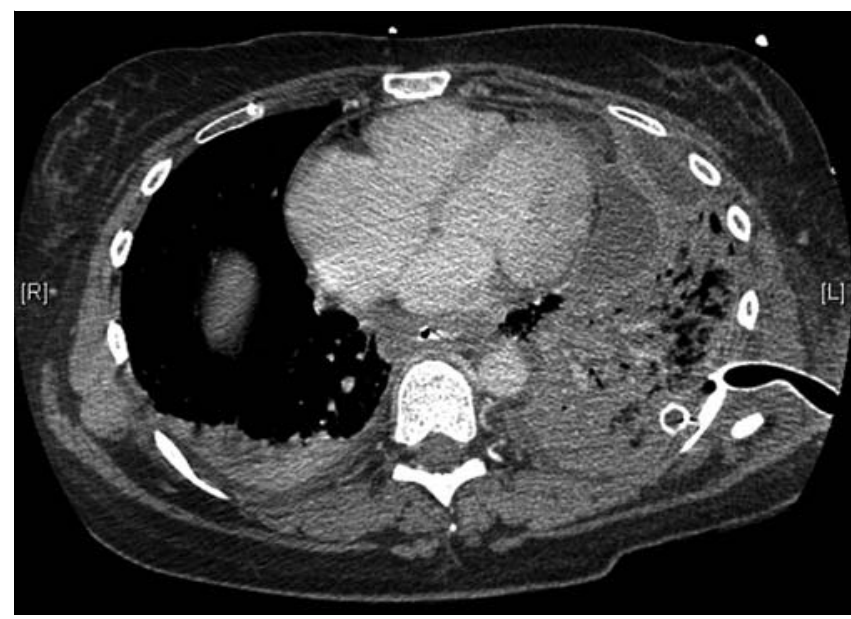

Fig. 2 Thoracic computed tomography (CT) scan showing empyema and mediastinitis with a surgical thoracic drain.

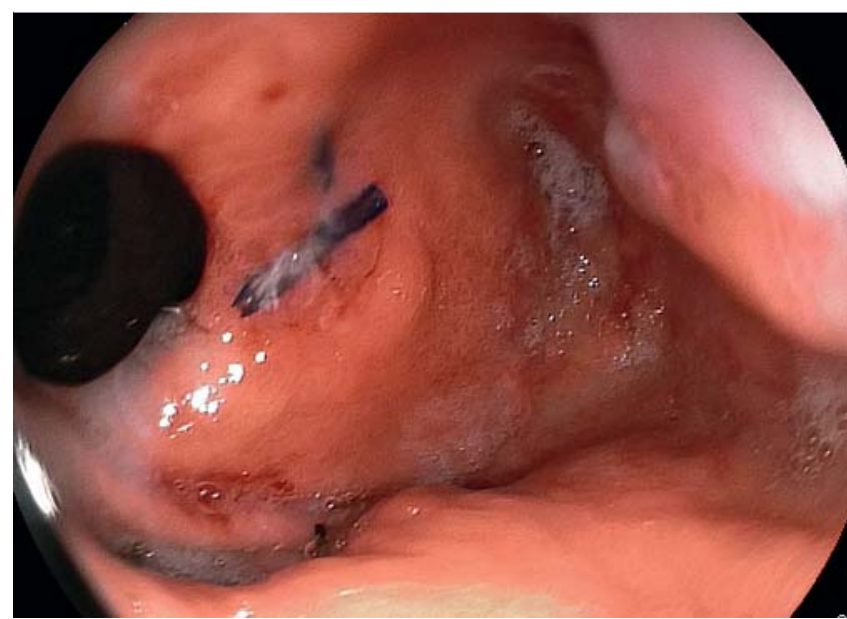

Fig. 3 Endoscopic image of the gastric pouch showing inadequate placement of fasteners around the gastrojejunostomy. 


\section{J. I. Lenz ${ }^{1}$, G. J. Hubens ${ }^{2}$, P. A. Pelck-} mans $^{1}$, T. G. Moreels ${ }^{1}$

1 Division of Gastroenterology and Hepatology, Antwerp University Hospital, Antwerp, Belgium

2 Division of Abdominal, Pediatric and Reconstructive Surgery, Antwerp University Hospital, Antwerp, Belgium

\section{References}

1 Thompson CC, Slattery J, Bunddga ME, Lautz $D B$. Peroral endoscopic reduction of dilated gastrojejunal anastomosis after Roux-en-Y gastric bypass: a possible new option for patients with weight regain. Surg Endosc 2006; 20: $1744-1748$

2 Elder KA, Wolfe BM. Bariatric surgery: a review of procedures and outcomes. Gastroenterology 2007; 132: 2253-2271

3 Mikami D, Needleman B, Narula V et al. Natural orifice surgery: initial US experience utilizing the StomaphyX device to reduce gastric pouches after Roux-en-Y gastric bypass. Surg Endosc 2010; 24: 223-228

4 Gibson S. Endoscopic solutions receives FDA clearance for first transoral surgical product: StomaphyXTM gets clearance first (April 2, 2007). Available from http://www. egseurope.com Accessed: November 15, 2010

5 Leitman IM, Virk CS, Avgerinos DV et al. Early results of trans-oral endoscopic plication and revision of the gastric pouch and stoma following Roux-en-Y gastric bypass surgery. JSLS 2010; 14: 217-220

\section{Bibliography}

DOI $10.1055 / \mathrm{s}-0030-1256258$

Endoscopy 2011; 43: E173-E174

(c) Georg Thieme Verlag KG Stuttgart · New York . ISSN 0013-726X

\section{Corresponding author}

\section{T. Moreels}

Antwerp University Hospital

Division of Gastroenterology and Hepatology

Wilrijkstraat 10

B-2650 Antwerp

Belgium

Fax: +32-3-8214478

tom.moreels@uza.be 